PROCEEDINGS OF THE

AMERICAN MATHEMATICAL SOCIETY

Volume 127, Number 4, April 1999, Pages 977-983

S 0002-9939(99)04790-5

\title{
ON ZEROS OF CHARACTERS OF FINITE GROUPS
}

\author{
DAVID CHILLAG
}

(Communicated by Ronald M. Solomon)

Dedicated to Avinoam Mann on the occasion of his 60th birthday

\begin{abstract}
We present several results connecting the number of conjugacy classes of a finite group on which an irreducible character vanishes, and the size of some centralizer of an element. For example, we show that if $G$ is a finite group such that $G \neq G^{\prime} \neq G^{\prime \prime}$, then $G$ has an element $x$, such that $\left|C_{G}(x)\right| \leq$ $2 m$, where $m$ is the maximal number of zeros in a row of the character table of $G$. Dual results connecting the number of irreducible characters which are zero on a fixed conjugacy class, and the degree of some irreducible character, are included too. For example, the dual of the above result is the following: Let $G$ be a finite group such that $1 \neq Z(G) \neq Z_{2}(G)$; then $G$ has an irreducible character $\chi$ such that $\frac{|G|}{\chi^{2}(1)} \leq 2 m$, where $m$ is the maximal number of zeros in a column of the character table of $G$.
\end{abstract}

\section{INTRODUCTION}

Let $\chi$ be an irreducible ordinary non-linear character of a finite group $G$. A well-known theorem of Burnside ([7], p. 40) states that $\chi$ has a zero on $G$, that is, an $x \in G$ with $\chi(x)=0$. As far as we know, the best lower bound on the number of zeros of such a $\chi$ were obtained by Zhmud in [11]. Zhmud also studied the subgroup generated by the zeros of a character (e.g. [12]). Groups that have an irreducible character that vanishes on exactly one class were studied by Zhmud in [13], and those having an irreducible character vanishing on all but two classes were studied by Gagola in [4].

Our purpose here is to show that if an irreducible character of a finite group vanishes on exactly $m$ conjugacy classes, then either the group has an element whose centralizer size is at most $2 m$, or the group and the character are restricted in some sense. Later on we will discuss dual results on the number of irreducible characters vanishing on a fixed conjugacy class. In fact, we present two simple observations (Theorems 1.1 and 1.3) and the rest of the results are consequences of these. Our first observation is:

Theorem 1.1. Let $G$ be a finite non-abelian group such that $G \neq G^{\prime}$, and let $\chi$ be a non-linear irreducible character of $G$. Let $m$ be the number of conjugacy classes of $G$ on which $\chi$ vanishes. Then one of the following holds:

Received by the editors August 1, 1997.

1991 Mathematics Subject Classification. Primary 20Cxx.

(C)1999 American Mathematical Society 
1. $G$ has an element $x$ such that $\left|C_{G}(x)\right| \leq 2 m$. In fact, one can choose such an $x$ from any conjugacy class of maximal size among the classes on which $\chi$ vanishes.

2. $\phi=\chi_{G^{\prime}} \in \operatorname{Irr}\left(G^{\prime}\right)$ and $\phi^{G}=\chi \sum\{\lambda \mid \lambda \in \operatorname{Irr}(G), \lambda(1)=1\}$. Furthermore, the set $\{\chi \lambda \mid \lambda \in \operatorname{Irr}(G), \lambda(1)=1\}$ consists of exactly $\left|G: G^{\prime}\right|$ extensions of $\phi$, which are all the extensions of $\phi$. In particular, $\phi$ is not linear and $G^{\prime \prime} \neq 1$.

The next result is:

Theorem 1.2. Let $G$ be a finite non-abelian group such that $G \neq G^{\prime}$. For every $\chi \in \operatorname{Irr}(G)$, let $n(\chi)$ be the number of conjugacy classes of $G$ on which $\chi$ vanishes. Set $m=\max \{n(\chi) \mid \chi \in \operatorname{Irr}(G)\}$. Assume that $\left|C_{G}(x)\right|>2 m$ for all $x \in G$. Then all the following hold:

1. For all $\chi \in \operatorname{Irr}(G)$ we have $\chi_{G^{\prime}} \in \operatorname{Irr}\left(G^{\prime}\right)$, and the set $\{\chi \lambda \mid \lambda \in \operatorname{Irr}(G), \lambda(1)=$ $1\}$ consists of exactly $\left|G: G^{\prime}\right|$ extensions of $\chi_{G^{\prime}}$, which are all the extensions of $\chi_{G^{\prime}}$.

2. Each $\varphi \in \operatorname{Irr}\left(G^{\prime}\right)$ is of the form $\chi_{G^{\prime}}$ for some $\chi \in \operatorname{Irr}(G)$.

3. $G^{\prime \prime}=G^{\prime}$.

4. Every $G^{\prime}$-conjugacy class is a $G$-conjugacy class.

5. Every non-linear irreducible character of $G$ vanishes on at least one conjugacy class outside $G^{\prime}$ and on at least one conjugacy class inside $G^{\prime}$.

The proofs of the above theorems, as well as consequences for metabelian groups, solvable groups and groups with irreducible characters vanishing on exactly one class, can be found in Section 2.

The main dual observation is the following $\left(\operatorname{here~}_{G}(u)\right.$ is the $G$-conjugacy class of the element $u$ of $G$ ):

Theorem 1.3. Let $G$ be a finite non-abelian group such that $Z(G) \neq 1$, and let $C$ be a non-central conjugacy class of $G$. Let $m$ be the number of irreducible characters of $G$ vanishing on $C$. Then one of the following holds:

1. $G$ has an irreducible character $\chi$ such that $\frac{|G|}{\chi^{2}(1)} \leq 2 m$. In fact, $\chi$ may be chosen to be an irreducible character of maximal degree among the irreducible characters vanishing on $C$.

2. If $c \in C$, then $\left|\mathrm{cl}_{G / Z(G)}(c Z(G))\right|=|C|$. In particular, $G / Z(G)$ is not abelian.

The proof and consequences which are "dual" to the ones in Section 2 can be found in Section 3

Our notation is standard and taken mainly from [7]. The value of the class function $f$ on the conjugacy class $C$ will be denoted by $f(C)$. Also, we will denote the set of all linear characters of the group $G$ by $\operatorname{Lin}(G)$.

\section{The number of CONJUGACY ClASSES ON WHICH AN IRREDUCIBLE CHARACTER VANISHES}

Proof of Theorem 1.1. Let $D_{1}, D_{2}, \ldots, D_{m}$ be all the conjugacy classes of $G$ on which $\chi$ vanishes. Then $\operatorname{Lin}(G)$ acts on $\operatorname{Irr}(G)$ by multiplication. Let $O=\{\chi \lambda \mid$ $\lambda \in \operatorname{Lin}(G)\}$ be the orbit of $\chi$ in this action and set $r=\left|G: G^{\prime}\right|=|\operatorname{Lin}(G)|$. We now deal with two cases.

Case 1: $|O|<r$. Then, $\chi$ is fixed by some $\lambda \in \operatorname{Lin}(G)-\left\{1_{G}\right\}$, that is, $\lambda \chi=\chi$. Set $U=\bigcup_{i=1}^{m} D_{i}$; then $\operatorname{ker}(\lambda) \supseteq G-U$. So if $g \in G-U$, then $\lambda(g)=1$. Since 
$\left[\lambda, 1_{G}\right]=0$ we get that:

$$
|G|=\sum_{g \in G} 1-\sum_{g \in G} \lambda(g)=\sum_{x \in U}(1-\lambda(x))=\sum_{i=1}^{m}\left|D_{i}\right|\left(1-\lambda\left(D_{i}\right)\right) .
$$

So, if $D \in\left\{D_{1}, D_{2}, \ldots, D_{m}\right\}$ is of maximal size, then $|G| \leq|D| \sum_{i=1}^{m}\left|\left(1-\lambda\left(D_{i}\right)\right)\right| \leq$ $2 m|D|$. Let $d \in D$; then $\left|C_{G}(d)\right| \leq 2 m$ and so claim 1 holds.

Case 2: $|O|=r$. Write $\chi_{G^{\prime}}=e\left(\varphi_{1}+\varphi_{2}+\cdots+\varphi_{s}\right)$, where $\varphi_{1}, \varphi_{2}, \ldots, \varphi_{s}$ are the distinct Clifford conjugates of $\varphi=\varphi_{1}$ with $\varphi \in \operatorname{Irr}\left(G^{\prime}\right)$. By the Frobenius reciprocity, $\left[\varphi^{G}, \chi\right]=e$. Let $\lambda \in \operatorname{Lin}(G)$; then $\chi \lambda(x)=\chi(x)$ for all $x \in G^{\prime}$ and since $\varphi^{G}$ vanishes on $G-G^{\prime}$ we get that $\left[\varphi^{G}, \chi \lambda\right]=\left[\varphi^{G}, \chi\right]=e$. Thus $\chi \lambda$ is a constituent of $\varphi^{G}$ with multiplicity $e$, for all $\lambda \in \operatorname{Lin}(G)$. Now, $O$ is a regular orbit under $\operatorname{Lin}(G)$ so $\sigma=e \chi \sum_{\lambda \in \operatorname{Lin}(G)} \lambda$ is a constituent of $\varphi^{G}$. Comparing degrees we get:

$$
r \varphi(1)=\varphi^{G}(1) \geq e \chi(1) r=e \cdot e s \varphi(1) \cdot r \quad \text { which implies that } \quad e^{2} s \leq 1 .
$$

Thus $e=s=1$, so $\chi_{G^{\prime}}=\varphi \in \operatorname{Irr}\left(G^{\prime}\right)$. Furthermore $\sigma(1)=\chi(1) r=\varphi(1) r=\varphi^{G}(1)$ and hence $\varphi^{G}=\chi \sum_{\lambda \in \operatorname{Lin}(G)} \lambda$ which is another claim of 2 .

Since $\lambda_{G^{\prime}}=1_{G^{\prime}}$ for all $\lambda \in \operatorname{Lin}(G)$, we get that $(\chi \lambda)_{G^{\prime}}=\chi_{G^{\prime}}=\varphi$, and so every element of $O$ is an extension of $\varphi$. Next, if $\theta \in \operatorname{Irr}(G)$ is an arbitrary extension of $\varphi$, then $1=\left[\theta_{G^{\prime}}, \varphi\right]_{G^{\prime}}=\left[\theta, \varphi^{G}\right]$. But, $\varphi^{G}$ is the sum of the irreducible elements of $O$, and so $\theta \in O$. Finally, as $\varphi(1)=\chi(1)>1, \varphi$ is non-linear and so $G^{\prime \prime} \neq 1$.

Remark 2.1. The above proof shows that equality in claim 1 holds if and only if $\operatorname{ker}(\lambda)=G-\bigcup_{i=1}^{m} D_{i}$ and all elements of $\left\{D_{1}, D_{2}, \ldots, D_{m}\right\}$ have the same size $\frac{|G|}{2 m}$. So $|G: \operatorname{ker}(\lambda)|=2$ and $\bigcup_{i=1}^{m} D_{i}$ is the non-identity coset of $\operatorname{ker}(\lambda)$.

Corollary 2.2. Let $G$ be a finite non-abelian metabelian group. Let $s$ be the minimal size of a centralizer of an element of $G$. Then every non-linear irreducible character of $G$ vanishes on at least $\frac{s}{2}$ conjugacy classes.

Proof. Let $\chi$ be a non-linear irreducible character of $G$ and let $m$ be the number of conjugacy classes on which $\chi$ vanishes. Since $G^{\prime} \neq G$ and $G^{\prime \prime}=1$, Theorem 1.1 states that $\left|C_{G}(x)\right| \leq 2 m$ for some $x \in G$. So $s \leq\left|C_{G}(x)\right| \leq 2 m$ implies that $m \geq \frac{s}{2}$.

We need the following easy lemma.

Lemma 2.3. If an element of a finite non-abelian group $G$ has a centralizer of size 2, then $G$ is a Frobenius group with a complement of order 2 and an abelian odd-order kernel.

Proof. Clearly, $|G|>2$. Let $x \in G$ be such that $\left|C_{G}(x)\right|=2$. As $1 \neq x \in C_{G}(x)$, $x$ has order 2. Let $S \in \operatorname{Syl}_{2}(G)$ be such that $x \in S$. If $x \notin Z(S)$, then $\langle Z(S), x\rangle$ is a group of order at least four which is contained in $C_{G}(x)$, a contradiction. Thus $x \in Z(S)$ so that $S \subseteq C_{G}(x)$ forcing $|S|=2$. Then $G$ has a normal subgroup $O$ of index 2, and by assumption $x$ acts on $O$ without fixed points. It follows that $G$ is a Frobenius group with $S$ a complement and $O$ the Frobenius kernel. It is well known that if the complement has even order, the kernel is abelian.

Corollary 2.4. Let $G$ be a finite non-abelian group such that $G \neq G^{\prime}$. Let $\chi$ be a non-linear irreducible character of $G$ that vanishes on exactly one conjugacy class of $G$. Then one of the following holds: 
1. $G$ is a Frobenius group with a complement of order 2 and an abelian odd-order kernel.

2. $\phi=\chi_{G^{\prime}} \in \operatorname{Irr}\left(G^{\prime}\right)$ and $\phi^{G}=\chi \sum\{\lambda \mid \lambda \in \operatorname{Irr}(G), \lambda(1)=1\}$. In particular, $\phi$ is not linear and $G^{\prime \prime} \neq 1$.

Proof. By Theorem 1.1, either $G$ has an element whose centralizer has size 2, in which case claim 1 holds by Lemma 2.3, or claim 2 is true.

Remark 2.5. We note that the class upon which $\chi$ vanishes in Corollary 2.4 is either outside or inside $G^{\prime}$, and these two cases correspond exactly to the two conclusions of Corollary 2.4. Compare Corollary 2.4 with [11] and [13].

Proof of Theorem 1.2. 1. Let $\chi \in \operatorname{Irr}(G)$. If $\chi \in \operatorname{Lin}(G)$, then $\chi_{G^{\prime}}=1_{G^{\prime}} \in \operatorname{Irr}(G)$, so assume that $\chi \notin \operatorname{Lin}(G)$. If there exists an $x \in G$ such that $\left|C_{G}(x)\right| \leq 2 n(\chi)$; then $2 m<\left|C_{G}(x)\right| \leq 2 n(\chi)$ implies that $m<n(\chi)$ contradicting the definition of $m$. Thus no such $x$ exists and so by Theorem $1.1, \chi_{G^{\prime}} \in \operatorname{Irr}\left(G^{\prime}\right)$ and $\{\chi \lambda \mid \lambda \in$ $\operatorname{Irr}(G), \lambda(1)=1\}$ consists of exactly $\left|G: G^{\prime}\right|$ extensions of $\chi_{G^{\prime}}$, which are all the extensions of $\chi_{G^{\prime}}$.

2. Let $\varphi \in \operatorname{Irr}\left(G^{\prime}\right)$; then $\left[\varphi^{G}, \chi\right] \neq 0$ for some $\chi \in \operatorname{Irr}(G)$. Then $\left[\chi_{G^{\prime}}, \varphi\right]_{G^{\prime}} \neq 0$ and claim 1 implies that $\chi_{G^{\prime}}=\varphi$.

3. Let $\varphi \in \operatorname{Lin}\left(G^{\prime}\right)$; then by claim $2, \varphi=\chi_{G^{\prime}}$ for some $\chi \in \operatorname{Irr}(G)$ so that $\chi$ is linear. But then $\varphi=\chi_{G^{\prime}}=1_{G^{\prime}}$, so $\operatorname{Lin}\left(G^{\prime}\right)=\left\{1_{G^{\prime}}\right\}$ implying that $G^{\prime \prime}=G^{\prime}$.

4. This follows from Brauer's permutation lemma ([7], p. 93). Since all elements of $\operatorname{Irr}\left(G^{\prime}\right)$ are invariant under $G$ (by claim 2), all the conjugacy classes of $G^{\prime}$ are $G$-invariant as well. Now the claim follows.

5. By $4, G$ fixes every conjugacy class of $G^{\prime}$. Thus a theorem of Gallagher ([3]) implies that each non-linear irreducible character $\chi$ of $G$ has zeros outside $G^{\prime}$. As $\chi_{G^{\prime}} \in \operatorname{Irr}\left(G^{\prime}\right)$ is non-linear, it is well known that it has a zero in $G^{\prime}$ as well.

An obvious corollary is:

Corollary 2.6. Let $G$ be a finite non-abelian group such that $G \neq G^{\prime} \neq G^{\prime \prime}$ (in particular, this holds for $G$ solvable). For every $\chi \in \operatorname{Irr}(G)$, let $n(\chi)$ be the number of conjugacy classes of $G$ on which $\chi$ vanishes. Set $m=\max \{n(\chi) \mid \chi \in \operatorname{Irr}(G)\}$. Then $G$ has an element $x$ such that $\left|C_{G}(x)\right| \leq 2 m$.

The proof of the following consequence uses the classification of the finite simple groups in the case $G=G^{\prime}$. The classification is not needed if $G \neq G^{\prime}$.

Proposition 2.7. Let $G$ be a finite non-abelian group. Assume that each $\chi \in$ $\operatorname{Irr}(G)$ vanishes on at most one conjugacy class. Then $G$ is a Frobenius group with a complement of order 2 and an abelian odd-order kernel.

Proof. Assume first that $G \neq G^{\prime}$. With the notation of Theorem 1.2, here $m=1$. If $\left|C_{G}(x)\right|>2$ for all $x \in G$, then Theorem 1.2 states that each non-linear irreducible character of $G$ vanishes on at least two classes (one in $G^{\prime}$ and one outside $G^{\prime}$ ), a contradiction. Hence $\left|C_{G}(x)\right|=2$ for some $x \in G$ and the result follows from Lemma 2.3. We now show that $G=G^{\prime}$ is impossible.

Assume the contrary, that is, $G=G^{\prime}$. By assumption, $G$ has a normal subgroup $N$, such that $X=G / N$ is a non-abelian simple group. Let $\chi \in \operatorname{Irr}(G / N)$ be nonlinear. If $\chi(a N)=\chi(b N)=0$, then $\chi(a)=\chi(b)=0$ and by assumption $a$ and $b$ are conjugate in $G$, so $a N$ and $b N$ are conjugate in $X$. It follows that every non-linear irreducible character of $X$ vanishes on exactly one conjugacy class of $X$. We will 
now show that there exists $\chi \in \operatorname{Irr}(X)$ vanishing on at least two conjugacy classes. This will provide the needed contradiction. Suppose first that $X$ has a 2-block of defect zero. Then by Theorem 8.17, p. 133 of [7], $X$ has an irreducible character vanishing on every element of even order. It follows that $X$ has exactly one class of element of even order and this must be the class of involutions. In particular a Sylow 2-subgroup $S$ of $X$ is elementary abelian and if $g \in S$, then $C_{G}(s)=S$ (otherwise there would be elements of even order which are not involutions). By [9] (see remark at the end of the proof) $X \simeq L_{2}\left(2^{n}\right)$ for some $n$ (as $S_{z}\left(2^{n}\right)$ has non-abelian Sylow 2 -subgroups). So $X$ has an irreducible character vanishing on at least two conjugacy classes (see for example pp. 1829-1830 of [8]), a contradiction. So $X$ has no 2-block of defect zero. Now [10] implies that $X$ is one of the following groups: $A_{n}, M_{12}, M_{22}, M_{24}, J_{2}, H S, S u z, R u, C 1, C 3, B M$. Each of the sporadic groups from this list as well as $A_{n}, n \leq 9$, has an irreducible character vanishing on more than one conjugacy class, as can be seen from [2]. So $X=A_{n}, n \geq 10$. Then $X$ has an irreducible character $\theta$ of degree $n-1$ such that for $g \in X, \theta(g)$ is the number of fixed points of $g$ minus 1 . Let $x=(1,2, \ldots, n-4)(n-3, n-2, n-1)(n)$ and $y=(1,2, \ldots, n-6)(n-5, n-4, n-3, n-2, n-1)(n)$. If $n$ is odd, then $x, y \in A_{n}, \theta(x)=\theta(y)=0$ and $x$ and $y$ are not conjugate. So $n$ is even. Then $z=(1,2, \ldots, n-1)(n)$ and $w=(1,2, \ldots, n-5)(n-4, n-3)(n-2, n-1)(n)$ are two non-conjugate elements of $A_{n}$ with $\theta(z)=\theta(w)=0$, a contradiction.

Remark 2.8. 1. In the proof of Proposition 2.7 we had the situation in which $X$ is a simple group with an abelian Sylow 2-subgroup $S$ such that $S=C_{G}(x)$ for all $x \in S-\{1\}$. We quoted [9] to conclude that $X \simeq L_{2}\left(2^{n}\right)$. It was pointed out by the referee and the editor that a very elegant and elementary proof of this result can be found in [5], and that in fact this result goes back to Burnside.

2. Let $k$ be the number of conjugacy classes of the group $G, C$ be a conjugacy class of maximal size, and $c \in C$. Then clearly $\left|C_{G}(c)\right| \leq k$. So the results of the existence of a centralizer of size at most $2 m$ gives new information only if $m$ is small relative to $k\left(m<\frac{k}{2}\right)$.

3. Y. Berkovich pointed out that if $G$ is an arbitrary non-abelian $p$-group, then every non-linear irreducible character $\chi$ vanishes on at least $n_{p}=p(p-1)$ conjugacy classes. If $G$ is not of maximal class, then $\chi$ vanishes on at least $n_{p}=p^{2}(p-1)$ conjugacy classes.

\section{The NUMBER OF IRREDUCIBLE CHARACTERS VANISHING ON A CONJUGACY CLASS}

The proof of Theorem 1.3 is kind of "dual" to that of Theorem 1.1.

Proof of Theorem 1.3. Set $Z=Z(G)$. Let $c \in C$ and let $\left\{\theta_{1}, \theta_{2}, \ldots, \theta_{m}\right\}$ be the set of all irreducible characters of $G$ vanishing on $C$. Next, $Z$ acts on the set of the conjugacy classes of $G$ by multiplication. Let $O=\{z C \mid z \in Z\}$ be the orbit of $C$ in this action. Recall that for every $\chi \in \operatorname{Irr}(G)$ we have that $\chi_{Z}=\chi(1) \lambda_{\chi}$ for some $\lambda_{\chi} \in \operatorname{Irr}(Z)$ (see [7], pp. 26-27), in particular $\operatorname{ker}\left(\lambda_{\chi}\right) \subseteq \operatorname{ker}(\chi)$. We now deal with two cases.

Case 1: $|O|<|Z|$. In this case $C$ is fixed by some $z \in Z-\{1\}$, that is, $z C=C$. So $z c$ is conjugate to $c$ and consequently $\chi(z c)=\chi(c)$ for all $\chi \in \operatorname{Irr}(G)$. So $\lambda_{\chi}(z) \chi(c)=\chi(c)$ for all $\chi \in \operatorname{Irr}(G)$. It follows that $I=\operatorname{Irr}(G)-\left\{\theta_{1}, \theta_{2}, \ldots, \theta_{m}\right\} \subseteq$ 
$\operatorname{Irr}\left(\frac{G}{\langle z\rangle}\right)$. Thus,

$$
\begin{aligned}
|G| & =\sum_{i=1}^{m} \theta_{i}^{2}(1)+\sum_{\chi \in I} \chi^{2}(1) \leq \sum_{i=1}^{m} \theta_{i}^{2}(1)+\sum_{\chi \in \operatorname{Irr}\left(\frac{G}{\langle z\rangle}\right)} \chi^{2}(1) \\
& \leq m \theta^{2}(1)+\left|\frac{G}{\langle z\rangle}\right| \leq m \theta^{2}(1)+\left|\frac{G}{2}\right|
\end{aligned}
$$

where $\theta \in\left\{\theta_{1}, \theta_{2}, \ldots, \theta_{m}\right\}$ is of maximal degree. So $\frac{|G|}{\theta^{2}(1)} \leq 2 m$ and claim 1 holds.

Case 2: $|O|=|Z|$. It is easy to see (e.g. Lemma 1.1 of [1]) that $\left|\mathrm{cl}_{G}(c)\right| \geq$ $\left|\mathrm{cl}_{Z}(c Z)\right|$. To get the inverse inequality we set $\mathrm{cl}_{G}(c)=\left\{c^{g_{1}}, c^{g_{2}}, \ldots, c^{g_{s}}\right\}$ where $\left\{g_{1}=1, g_{2}, \ldots, g_{s}\right\} \subseteq G$, and show that $c^{g_{1}} Z, c^{g_{2}} Z, \ldots, c^{g_{s}} Z$ are all distinct. Indeed, if $c^{g_{i}} Z=c^{g_{j}} Z$ for some $i \neq j$, then for some $v \in Z-\{1\}$, we have $c^{g_{i}}=c^{g_{j}} v=(c v)^{g_{j}}$ so that $\operatorname{cl}_{G}(c)=\operatorname{cl}_{G}(c v)=v \mathrm{cl}_{G}(c)$. Hence $v$ fixes cl $\mathrm{cl}_{G}(c)$ implying that $|O|<|Z|$, a contradiction.

Corollary 3.1. Let $G$ be a nilpotent group of class 2 and $a \in G-Z(G)$. Let $m$ be the number of irreducible characters of $G$ vanishing on a. Then $G$ has an irreducible character such that $\frac{|G|}{\chi^{2}(1)} \leq 2 m$.

Proof. Since $\left|\mathrm{cl}_{G}(a)\right|>1$ and $\left|\operatorname{cl}_{G / Z(G)}(a Z(G))\right|=1$, claim 2 of Theorem 1.3 cannot hold. So claim 1 holds.

Corollary 3.2. Let $G$ be a finite non-abelian group such that $Z(G) \neq 1$. For each $a \in G$ let $n(a)$ be the number of irreducible characters of $G$ vanishing on a and set $m=\max \{n(a) \mid a \in G\}$. Assume that $\frac{|G|}{\chi^{2}(1)}>2 m$ for all $\chi \in \operatorname{Irr}(G)$. Then for every $a \in G,\left|\mathrm{cl}_{G / Z(G)}(a Z(G))\right|=\left|\mathrm{cl}_{G}(a)\right|$. In particular $Z_{2}(G)=Z(G)$.

Proof. Set $Z=Z(G)$ and let $a \in G$. If $a \in Z$, then $\left|\operatorname{cl}_{G}(a)\right|=\left|\operatorname{cl}_{G / Z}(a Z)\right|=1$, so we may assume that $a \notin Z$. If $G$ has an irreducible character $\chi$ such that $\frac{|G|}{\chi^{2}(1)} \leq 2 n(a)$, then $\frac{|G|}{\chi^{2}(1)} \leq 2 m$, contradicting our assumption. Thus claim 1 of Theorem 1.3 does not hold. So claim 2 holds and so $\left|\mathrm{cl}_{G}(a)\right|=\left|\mathrm{cl}_{G / Z}(a Z)\right|>1$, as claimed. In particular $\left|\mathrm{cl}_{G / Z}(a Z)\right|=1$ if and only if $a Z=Z$ proving that $Z\left(\frac{G}{Z}\right)=1$, which is the second claim of the corollary.

An immediate corollary is:

Corollary 3.3. Let $G$ be a finite non-abelian group such that $1<Z(G)<Z_{2}(G)$. For each $a \in G$ let $n(a)$ be the number of irreducible characters of $G$ vanishing on $a$ and set $m=\max \{n(a) \mid a \in G\}$. Then $G$ has an irreducible character $\chi$ such that $\frac{|G|}{\chi^{2}(1)} \leq 2 m$.

\section{ACKNOWLEDGMENT}

I wish to thank Y. Berkovich, I. M. Isaacs, A. Mann and the referee for their helpful comments.

\section{REFERENCES}

1. D. Chillag and M. Herzog, On the length of the conjugacy classes of finite groups, J. Algebra 131 (1990), 100-125. MR 91c:20038

2. J. H. Conway, R. T. Curtis, S. P. Norton, R. A. Parker and R. A. Wilson, Atlas of Finite Groups, Clarendon Press, 1985. MR 88g:20025

3. P. X. Gallagher, Zeros of characters of finite groups, J. Algebra 4 (1966), 42-45. MR 34:252 
4. S. M. Gagola, Characters vanishing on all but two conjugacy classes, Pacific J. Math. 109 (1983), 363-385. MR 85e:20009

5. D. M. Goldsmith, Elements of order two in finite groups, Delta 4 (1974), 45-59.

6. B. Huppert, Endliche Gruppen I, Springer-Verlag, 1967. MR 37:302

7. I. M. Isaacs, Character Theory of Finite groups, Academic Press, 1976. MR 57:417

8. K. Ito (editor), Encyclopedic Dictionary of Mathematics, Vol. IV, Appendices and Indexes, second edition, MIT Press. MR 89b:00033

9. M. Suzuki, Two characteristic properties of (ZT)-groups, Osaka Math. J. 15 (1963), 143-150. MR 27:2548

10. W. Willems, Blocks of defect zero and degree problems, Proc. of Symposia in Pure Math. 47 (1987), 481-484. CMP 20:10

11. E. M. Zhmud, On estimates of the number of zeros of irreducible characters of a finite group, (Russian), Publ. Math. Debrecen 33 (1986), 125-146. MR 88a:20019

12. E. M. Zhmud, On Gallagher's theorems on zeros of groups characters (Russian), Publ. Math. Debrecen 37 (1990), 245-353. MR 91k:20016

13. E. M. Zhmud, On finite groups having an irreducible complex character with one class of zeros, Soviet Math. Dokl. 20 (1979), 795-797.

Department of Mathematics, Technion, Israel Institute of Technology, Haifa 32000 , ISRAEL

E-mail address: chillag@techunix.technion.ac.il 